\title{
Determination of the Sequence Specificity of XIAP BIR Domains by Screening a Combinatorial Peptide Library ${ }^{\dagger}$
}

\author{
Michael C. Sweeney, ${ }^{\star}$ Xianxi Wang, ${ }^{\S}$ Junguk Park, ${ }^{\natural}$ Yusen Liu, ${ }^{\S}$ and Dehua Pei ${ }^{*} \ddagger, \uparrow$
}

\section{Supporting Information}

Cell Culture and Transfection. HeLa cells were grown in a $37{ }^{\circ} \mathrm{C}$ humidified atmosphere containing 5\% $\mathrm{CO}_{2}$ in Dulbecco's modified Eagle's medium (Invitrogen, San Diego, CA) supplemented with 10\% fetal bovine serum (HyClone, Logan, UT). Cells (approximately $2 \times 10^{6}$ cells per $60-\mathrm{mm}$ plate) were transfected with either $4 \mu \mathrm{g}$ of pCMV-Sport6-caspase-10, or $4 \mu \mathrm{g}$ of pcDNA3, using LipofectAmine (Invitrogen) according to the manufacturer' recommendation. Forty-eight hours later, cells were harvested in a lysis buffer containing $10 \mathrm{mM}$ HEPES (pH 7.4), $50 \mathrm{mM} \beta$-glycerophosphate, 1\% Triton X-100, 10\% glycerol, 2 mM EDTA, 2 mM EGTA, $1 \mathrm{mM}$ DTT, $10 \mathrm{mM} \mathrm{NaF}, 1 \mathrm{mM} \mathrm{Na} \mathrm{VO}_{4}, 20 \mathrm{nM}$ microcystin-LR, $2 \mu \mathrm{M}$ leupeptin, $2 \mu \mathrm{M}$ aprotinin, and $1 \mathrm{mM}$ PMSF. The cells were incubated with the lysis buffer on ice for $15 \mathrm{~min}$ to facilitate protein extraction, collected using sterile scrapers, and then transferred into Eppendorf tubes for centrifugation. The soluble lysates were collected after centrifugation at 14,000 g for $10 \mathrm{~min}$ at 4 ${ }^{\circ} \mathrm{C}$.

Reagents. Recombinant human caspase-3 and caspase-10 were purchased from BioVision (Mountain View, CA). The caspase substrates, acetyl (Ac)-DEVD-7-Amino-4trifluoromethylcoumarin (AFC) and Ac-AEVD-AFC, were purchased from MP Biomedicals (Aurora, $\mathrm{OH}$ ).

Caspase Activity Assays. For caspase activity determination, $100-\mu \mathrm{L}$ reactions containing either $50 \mu \mathrm{g}$ of a cell lysate harvested from cells transfected with caspase-10 expression vector or the indicated amounts of recombinant caspase and $100 \mu \mathrm{M}$ specific caspase substrate were incubated at $37{ }^{\circ} \mathrm{C}$ for $60 \mathrm{~min}$. Capase-3 activity was determined using Ac-DEVD-AFC as a substrate. Caspase-10 activity was assayed using Ac-AEVD-AFC as a substrate. The fluorescence intensities of the samples were measured using $410 \mathrm{~nm}$ excitation and $505 \mathrm{~nm}$ emission with a SpectraMax M2 fluorescent plate reader (Molecular Devices, Sunnyvale, CA).

Statistics. Statistics were determined using Independent-samples T test (SPSS, Chicago, IL). 
Table S1. Potential BIR2-binding partners from Protein Information Resource database search.

\begin{tabular}{|c|c|}
\hline Protein & Motif \\
\hline Zinc Finger Protein 202 & MATAV \\
\hline Mitochondrial Ribosomal Protein S5 & MATAV \\
\hline Tripeptidyl Peptidase II & MATAA \\
\hline LAG1 Longevity Assurance Homolog 5 & MATAA \\
\hline Squamous Cell Carcinoma Antigen Recognized by T-Cells 3 (SART3) & MATAA \\
\hline Remodeling and Spacing Factor 1 & MATAA \\
\hline Procollagen (type III) N-Endopeptidase & MATAA \\
\hline POU Domain, Class 3, Transcription Factor 3 & MATAA \\
\hline WS $\beta$-Transducin Repeats Protein & MATAA \\
\hline Low-Density Lipoprotein B & MATAA \\
\hline N-Oct 3 & MATAA \\
\hline Nicastrin & MATAG \\
\hline Kallikrein 4 & MATAG \\
\hline RAB14 & MATAP \\
\hline Protein Kinase C, D2 type & MATAP \\
\hline Nedd4 Binding Protein 3 & MATAP \\
\hline Katanin p80 Subunit B1 & MATPV \\
\hline Translation Initiation Factor 3, Subunit $5 \varepsilon$ & MATPA \\
\hline GRINL1A Downstream Protein Gdown 4 & MATPA \\
\hline BCL2-like 2 Protein & MATPA \\
\hline ARNT2 Protein & MATPA \\
\hline ATP-Dependent RNA Helicase \#3 & MATPA \\
\hline Calponin Homology Domain Containing 1 & MATPG \\
\hline Phosphatidylinositol-4-phosphate 5-kinase type II $\alpha$ & MATPG \\
\hline PP13 & MATPP \\
\hline UXT Protein & MATPP \\
\hline Deoxycytidine Kinase & MATPP \\
\hline Zinc Finger Protein 268 & MATRV \\
\hline Gamma-Taxilin & MATRV \\
\hline Zinc Finger Transcription Factor BTEB2 & MATRV \\
\hline P120 & MATRG \\
\hline SH3PX1 Protein & MATKA \\
\hline Superoxide Dismutase & MATKA \\
\hline Kelch/Ankyrin Repeat Containing Cyclin A1 Interacting Protein (KARCA1) & MAVAV \\
\hline Septin 11 & MAVAV \\
\hline RNA Helicase & MAVAV \\
\hline SCAND2 Protein & MAVAV \\
\hline Pinin & MAVAV \\
\hline$\beta 1-$ Syntrophin & MAVAA \\
\hline Mitochondrial 28S Ribosomal Protein S32 & MAVAA \\
\hline Thyroid Hormone Receptor Interactor 4 & MAVAG \\
\hline $\mathrm{ABCD} 4$ & MAVAG \\
\hline Ubiquitin Specific Protease 11 & MAVAP \\
\hline COM Domain Containing 10 & MAVPA \\
\hline Putative Tumor Suppressor Gene 26 Protein & MAVPA \\
\hline THO Complex 3 & MAVPA \\
\hline Dachshund & MAVPA \\
\hline Phosphodiesterase 3A & MAVPG \\
\hline G Patch Domain Containing 3 & MAVPG \\
\hline PAP Associated Domain Containing 1 & MAVPG \\
\hline Dual-Specificity Tyrosine-Phosphorylation Regulated Kinase 1B (DYRK1B) & MAVPP \\
\hline
\end{tabular}




\begin{tabular}{|l|l|}
\hline MICAL-Like Isoform 2 & MAVPP \\
\hline Ethanolamine Kinase 2 & MAVPP \\
\hline GROS1-L Protein & MAVRA \\
\hline Integrin 34 Binding Protein & MAVRA \\
\hline Bagpipe homeobox 1 & MAVRG \\
\hline Solute Carrier Family 25 & MAVKV \\
\hline Dedicator of Cytokinesis 6 (DOCK6) & MAIAG \\
\hline Mitochondrial Serine Hydroxymethyltransferase & MAIRA \\
\hline L-Asparaginase & MARAV \\
\hline Centrosomal Protein of 72 kDa & MARAG \\
\hline Spire Homolog 2 & MARAG \\
\hline Leucine-Rich Repeats and Immunoglobulin-Like Domains 1 & MARPV \\
\hline Winged Helix Domain-Containing Protein & MARPV \\
\hline Albumin D-Box Binding Protein & MARPV \\
\hline Brain Acyl-CoA Hydrolase (BACH) & MARPG \\
\hline ARPG863 & MARPG \\
\hline Neuromedin B & MARRA \\
\hline Constitutive Androstane Receptor SV16 & MARRP \\
\hline SPRY Domain-Containing SOCS Box Protein SSB-3 & MARRP \\
\hline c-myb & MARRP \\
\hline V-myb myeloblastosis viral oncogene homologue & MARRP \\
\hline WD Repeat Domain 40A & MARKV \\
\hline aarF Domain Containing Kinase 1 & MARKA \\
\hline HSP70-2 & MAKAA \\
\hline RNA Processing Factor & MAKAG \\
\hline NIR1 & MAKAG \\
\hline Ankyrin Repeat Domain 2 & MAKAP \\
\hline Annexin VI & MAKPA \\
\hline Hexokinase 1 & MAKRA \\
\hline Zinc Finger Protein 479 & MAKRP \\
\hline Zinc Finger Protein 679 & MAKRP \\
\hline Flavin Containing Monooxygenase 4 & MAKKV \\
\hline
\end{tabular}


A

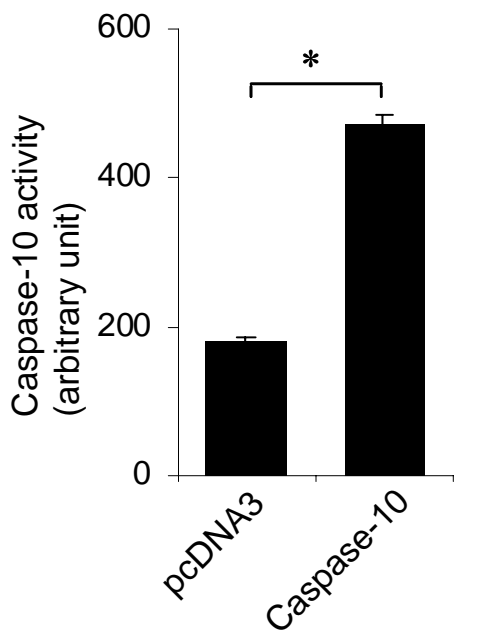

B

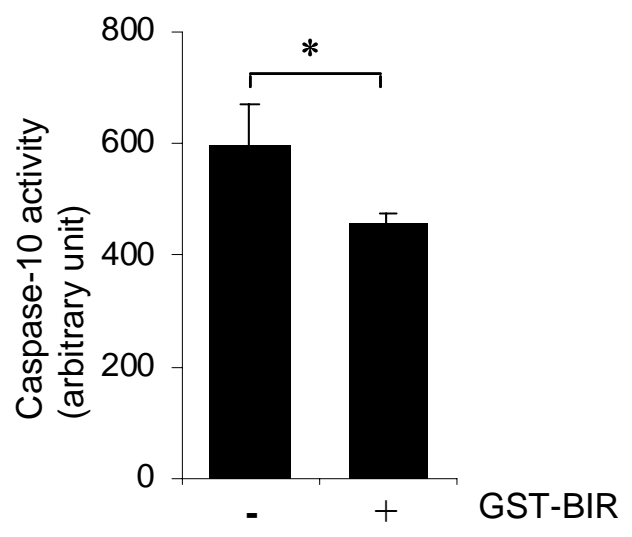

Figure S1. Inhibition of caspase-10d by XIAP BIR1-3 domains. HeLa cells $\left(2 \times 10^{6}\right.$ cells per plate) were transfected with a caspase-10 expression vector (pCMV-SPORT6-Caspase-10d, $4 \mu \mathrm{g}$ ) or an empty vector pcDNA3 $(4 \mu \mathrm{g})$. Caspase-10d activity in the lysates was measured by fluorometric assays using 96-well plates. The effect of BIR domains on caspase-10 activity was assessed by assaying caspase-10d in the presence of various amounts of GST-BIR1-3 fusion protein. (A) Caspase-10d activity in lysates isolated from HeLa cells transfected either with pCMV-SPORT6-casapse-10d or pcDNA3. (B) Effect of GST-BIR1-3 domains (7.5 $\mu \mathrm{M})$ on caspase-10d activity. Data represent the mean \pm SD from 3 independent experiments. *, $p<0.05$.

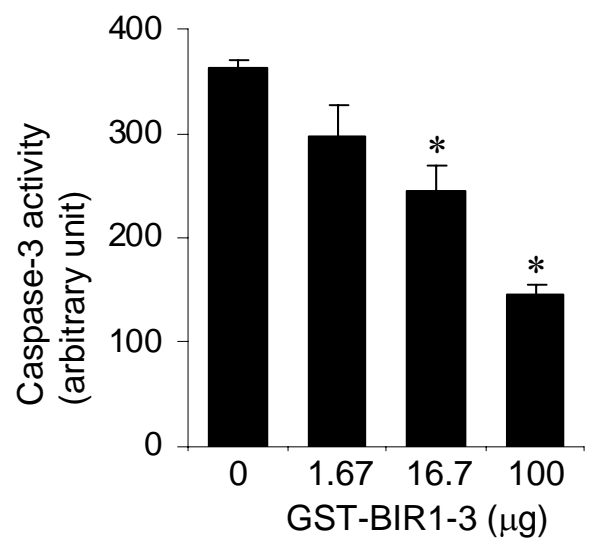

Figure S2. Inhibition of caspase-3 by GST-BIR1-3 fusion protein. The effect of XIAP BIR domains on caspase-3 activity was assessed by fluorometric assays in the presence of increasing amount of GST-BIR1-3 fusion protein (0-100 $\mu$ g or GST-BIR1-3/Caspase-3 molar ratio of 06000). Each reaction contained 0.5 unit of caspase-3 (BioVision). The specific activity of caspase-3 was $30 \mathrm{unit} / \mu \mathrm{g}$. Data represent the mean \pm SD from 3 independent experiments. *, $p<0.05$. 


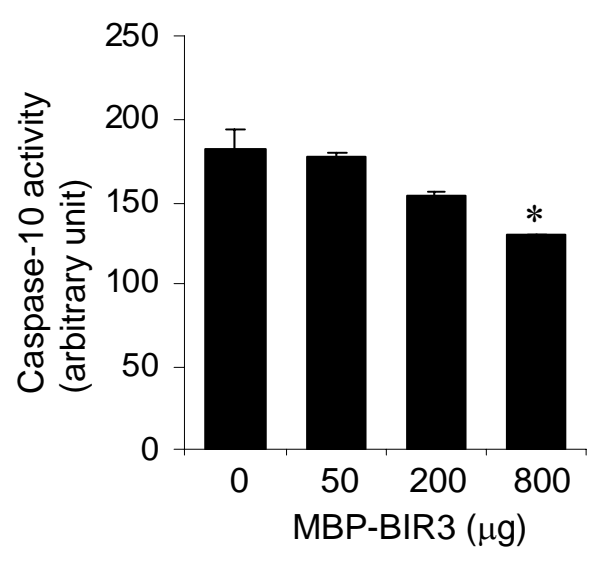

Figure S3. Inhibition of caspase-10d by the MBP-BIR3 protein. The graph depicts that the activity of recombinant caspase-10d in the absence or presence of varying amounts of MBPBIR3 fusion protein (0-800 $\mu \mathrm{g}$ or BIR3/caspase molar ratio of 0-12000). Caspase-10d activity was assessed by fluorometric assays in reactions containing 1 unit of caspase-10d (15 unit/ $\mu \mathrm{g})$. Values represent mean $\pm \mathrm{SD}$ from 3 independent experiments. ${ }^{*}, p<0.05$. 